\title{
Evaluation of industrial effluent and domestic sewage genotoxicity using Allium cepa bioassay
}

\author{
Rajesh Paul and Jashodeb Arjun* \\ Zoology Department Lumbding College, Lumbding, 782447, Assam
}

\section{ABSTRACT}

Living organisms including both plants and animals being exposed to polluted water bodies can be impacted. In current investigation the genotoxic effect of industrial effluent and domestic sewage of Barak valley region, Assam, India was investigated using both morphological and root chromosome assay on Allium cepa. The mean root lengths of onions exposed to different concentrations of the effluent and sewage (10\%, 25\% and 50\%) were measured for 3 consecutive days for 24, 48 and $72 \mathrm{hrs}$ and the results were compared. The mean root length was statistically evaluated by the analysis of variance. There was both significant increase and decrease in root length among the exposed onion bulbs. Total aberrations increased significantly as concentration increased $(\mathrm{p}<0.05)$. Both effluent and sewage samples were recorded to cause harmful damages in the exposed onion test samples. These results demonstrated that the Allium test is a useful screening test for the evaluation of toxicity caused by sewage and effluent samples not only at the morphological level but also at the cytogenetic level; and hence pollution in waster bodies in a major cause of concern. Thus, sincere measures should be undertaken regarding the direct disposable of industrial effluents and domestic sewage and protection of water bodies including its flora and fauna.

KEY WORDS: PAPER MILL EFFLUENTS, DOMESTIC SEWAGE, ALLIUM CEPA TEST

ARTICLE INFORMATION:

*Corresponding Author: jashodeb@gmail.com

Received $19^{\text {th }}$ July, 2018

Accepted after revision $17^{\text {th }}$ Sep, 2018

BBRC Print ISSN: 0974-6455

Online ISSN: 2321-4007 CODEN: USA BBRCBA

\%... Thomson Reuters ISI ESC / Clarivate Analytics USA and

Crossref Indexed Journal

NAAS Journal Score 2018: 4.31 SJIF 2017: 4.196

$\odot$ A Society of Science and Nature Publication, Bhopal India 2018. All rights reserved.

Online Contents Available at: http//www.bbrc.in/

DOI: $10.21786 / \mathrm{bbrc} / 11.3 / 19$ 


\section{INTRODUCTION}

Over two third of Earth's surface is covered by water; less than a third is taken up by land. As Earth's population continues to grow, people are putting ever-increasing pressure on the planet's water resources .In a sense, our oceans, rivers and other inland waters are being squeezed by human activities, not so that they take up less room, but so their quality is reduced. Poorer water quality leads to pollution in water bodies. Water pollution nowadays is a major global problem. It requires ongoing evaluation and revision of water resource policy at all levels. It has been recorded that water pollution is the leading worldwide cause of death and diseases and it accounts for the death of more than 14,000 people daily. India and China are the two countries with high levels of water pollution: An estimated 580 people in India die of water pollution related illness including water borne diseases. Water pollution results in contamination of water bodies like rivers, lakes, aquifer, etc. due to human activities, (Bennet, 1997, Prasad and Rao, 2010. Bakare et al, 2017, Anacleto et al, 2017).

Polllution in water bodies occurs when contaminants gets introduced into the natural environment. For example, releasing inadequately treated waste water into natural water bodies leading to degradation of aquatic ecosystem. This in turn can cause public health problems for people living in downstream as people are using this polluted water for regular domestic activities like bathing, washing, drinking, irrigation, etc. Researches have revealed that the sources of water pollution may be point sources and non-point sources. Point sources have an identifiable cause as storm drain, waste water treatment plant or streams. Non-point sources are more diffuse like agricultural runoff, (Zaiad, 2010). With the increasing development of industrial resources the risk of water pollution has also increased. Not only the industrial resources but also the inadequate system of dumping of municipal sewage has resulted in pollution of water bodies and its aquatic ecosystem including both aquatic flora and fauna.

Researches till date have revealed that dissolved contaminants in both effluents and sewages when exposed to water bodies not only harms plant growth but also forces plants to absorb dangerous chemicals and pollutants which gets passed to animals and human through consumption and other modes (Sik et al, 2009). In a work done on the variable actions of Alium sepa and its usage as a bio indicator of cadmium toxicity, plants were exposed to increasing concentrations of cadmium, where cadmium was observed to cause inhibition of root and leaves growth and elongation which serve as a tool for characterizing the bio indication of cadmium exposure in waste and effluent condition, (Bakare et al, 2017).
The direct application of industrial sludges were recorded to harm the local biota in an investigation where the genotoxicity of industrial sludges was assessed using various plant including A.cepa where A.cepa test was found to be effective in detection of damages (Anacleto et al, 2017).

Studies about phytotoxic effects of waste waters and effluents started in 1970s where researches were conducted taking sugar cane, eucalyptus, Triticum aestivum, Brassica campastris, Sorghum, rice, and many more. Allium cepa is the largest genus of petaloid monocotyledons, containing hundreds of species naturally distributed in temperate climates of the northern hemisphere (Koçyi ğit \& Özhatay, 2010). This test has important advantages (Zegura, 2009) and has been used from many years in investigating physical and chemical mutagenesis and cytogenetic effects in mitotic cell division. Allium cepa is important since it is an excellent model in-vivo, where the roots grow in direct contact with the substance of interest enabling possible damage to the DNA of eukaryotes to be predicted. It is advantageous to use the Allium cepa test system since its main component is a vascular plant, making it an evaluating environmental pollutant , detecting mutagens (Gupta et al, 2009).

The present investigation was designed to examine the level of morphological and genotoxic damages caused by industrial effluents as well as domestic sewages on Allium cepa so that proper safety measures can be taken not only for the protection of water quality but also preventive measures can be taken against the damages caused to aquatic ecosystem prior to exposure of effluents and sewage.

\section{MATERIALS AND METHODS}

For present investigation raw paper mill effluent sample was collected from the outlet pipes in the local river Barak of the valley. The domestic sewage which was selected for comparative analysis was collected from Silchar, Municipal drainage system at Tarapur area, where all the debris and discharges of the whole locality have been found to be discharged. Both effluent and sewage samples were collected in plastic gallons, $\mathrm{pH}$ was measured and stored at $-20^{\circ} \mathrm{C}$ to prevent further microbial growth.

The common purple onion, Allium cepa $(2 \mathrm{n}=16)$ bulbs (1.5-2.0 cm diameter) used for this study was procured from organic farmers of almost equal weight age. The dried out scales were carefully removed leaving the ring of the root primordial intact (Fiskesjo, 2011). Then they were kept in moist condition to let root grow for three days, this help select onion with synchronous growth. For each test, $10 \mathrm{~A}$. cepa bulbs purchased from organic farmers were set up to produce roots in filtered and dechlorinized tap water for three days and then 
transferred to the test solutions. Tap water was previously filtered in a bio-activated coal filter to remove chlorine and its by-products commonly used for disinfecting drinking water. Three litres of water were aerated over a period of $24 \mathrm{~h}$ before filling the test tubes. For positive control mitomycin $\mathrm{C}$ was selected. Mitomycins are a family of azinidines containing natural products insulated from Streptomyces lavendulae. Mitomycin C is a potent DNA crosslinkers. A single cross link per genome has shown to be effective in killing bacteria. This is accomplished by reductive activities followed by $2 \mathrm{~N}$-Alkylation. Both alkylation are sequence specific for a guanine nucleotide sequence.

Three different concentrations of both the effluents and sewage were selected as 10\%, 25\% and 50\% for exposure through prior standardization. During the Allium cepa assay, all selected onions were exposed to the selected concentrations of effluent and sewage for $24 \mathrm{hrs}$, $48 \mathrm{hrs}$ and $72 \mathrm{hrs}$, respectively. The growth in roots were recorded till the third day of exposure in water and after that the variation in root growth were recorded after every $24 \mathrm{hrs}$ for next three consecutive days till $72 \mathrm{hrs}$ and the data were recorded and compared. For mitotic studies, growth inhibition tests were carried out for each sample, to find its toxicity level. After every $24 \mathrm{hrs}$ of exposure, 3 to 4 healthy root tips from each bulb were prepared for the microscopic slides.

The emerged root tips of the onion bulbs in the different concentration of sewage and effluent were fixed and macerated in a solution of 45\% acetic acid (9 parts) and $1 \mathrm{~N} \mathrm{HCl}$ ( 1 part) at $50{ }^{\circ} \mathrm{C}$ for $10 \mathrm{~min}$, followed by squashing in 2\% Acetocarmine stain for 15 minutes. The modified conventional Feulgen-squash method (Sharma and Dphil, 2012) was used to prepare permanent slides of root meristems. The root tips were put in 1 normal hydrochloric acid for five minutes to soften the tissue. The macerated and stained root tips were covered with cover slip and squashed. Minimum 3-4 Slides were prepared per bulb for microscopic observation. Approximately three thousand cells were examined per onion to remove the errors and classified according to the chromosomal aberrations presented including bridges, fragments and chromosome lagging.

Results were presented as Mean \pm SE where mean value was calculated from three individual readings of a particular set. ANOVA was performed to determine the level of significance from the set of onion bulbs. ANOVA was done using graph pad PRISM (Graph pad Inc., san Diego, CA, USA).

\section{RESULTS AND DISCUSSION}

Water pollution can be caused by a number of sources ranging from industrial resources and sewage treatment plants and factories to mining activities, paved roads and agricultural runoff. Such issues have become one of the biggest problems in many developing and developed countries. These pollutants when not treated properly, can cause mutagenic or toxic effects directly on humans, affecting human health, resulting in diseases like cancer, congenital malformations, and cardiovascular diseases (Grover Et Kauer, 2009). Siddiqui and his group (Siddiqui et al., 2011) have worked to validate plant-based tests for assessing the toxicity of water in India.

The Allium test is advantageous as genotoxicity screening assay, as Allium root cells posses the mixed function oxidase system which is capable of activating promutagens or genotoxic chemicals (Odeigah et al; 1997a). In the Allium test, inhibition of rooting and the appearance of stunted roots indicate retardation of growth and genotoxicty, while root wilting explains toxicity (Odeigah et al; 1997b). Both growth retardation and root wilting are accompanied by suppression of mitotic activity and remarkable chromosomal aberration. The present findings provides evidence that effluent and sewage inhibited root growth and caused growth retardation. The reason behind growth inhibition may be due to high rate of chemical oxygen demand which affected certain physiological processes leading to the disturbance in the balance between promoter and inhibitors of endogenous growth regulator (Gill and Saggoo, 2010).

Growth inhibition was most recorded at 50\% concentration along with a marked decrease in root length when compared with the control. This is usually accompanied by an increase in chromosome aberrations (Amin and Muzahid, 2009). The suppression of mitotic activity was often used in tracing cytotoxicity (Smaka-Kinel et al., 2013). In our study a decrease in the mitotic index was found as the concentration of effluent increased which indicates the cytotoxic effect of sewage and industrial effluent. Chrosomal aberration were observed to increase as the concentration of effluent and sewage increased. Among the chromosomal aberration observed, IN, EN and CF were most frequent in all concentrations band kept on increasing from concentrations of industrial effluent towards higher concentrations of sewage. Such findings are responsible for the completely decayed roots found in 25\% and 50\% concentration. The most common abnormalities were c-mitosis and disturbed metaphase. Sticky chromosomes and binucleated cells were recorded in noticeable amount. In addition to the above, at anaphase and telophase bridges, lagging chromosomes and irregular anaphase were also observed. The mitotic index in the root meristems grown in the negative control ranged from 17.3 to 19.8 .

Table 2 shows the mitotic index values in root meristems growth in different concentrations of effluent 

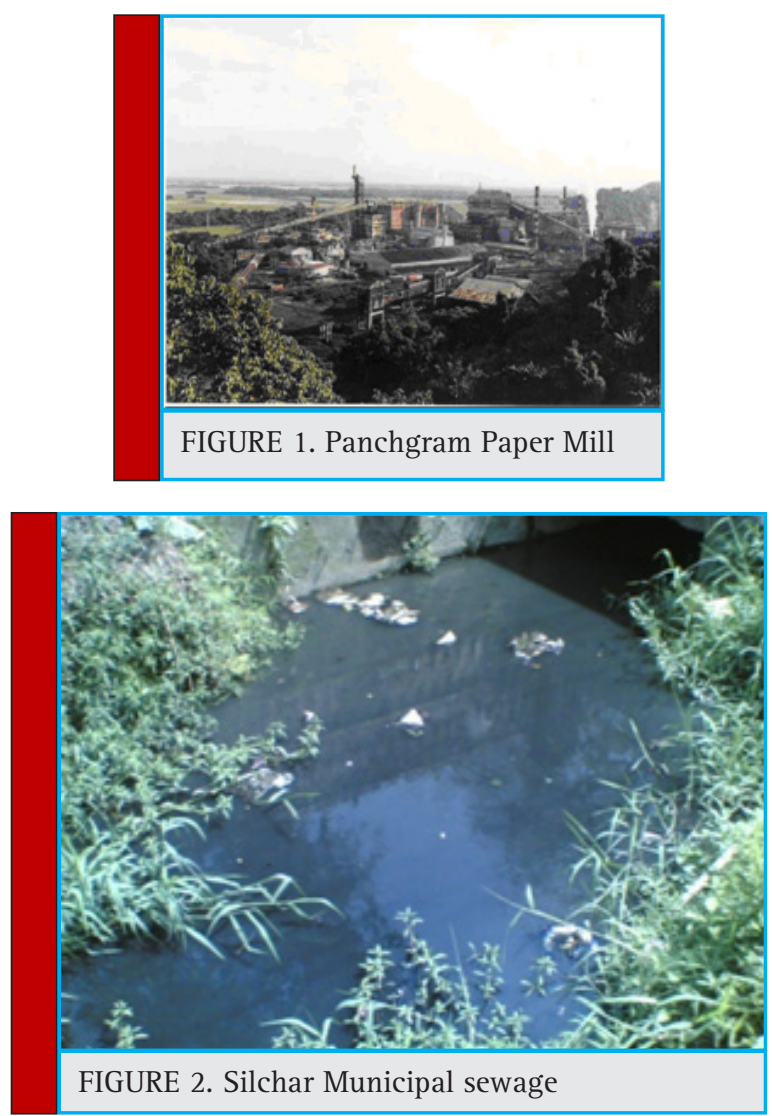

and sewage from the three concentrations of wastewater treatment. The decrease in the mitotic index of the root tips reached statistical significance only in the highest tested concentrations. The cytogenetic aberrations most commonly observed in anaphase-telophase cells were bridges, fragments and chromosome lagging. Aberrant mitotic cells were counted and expressed as mean and

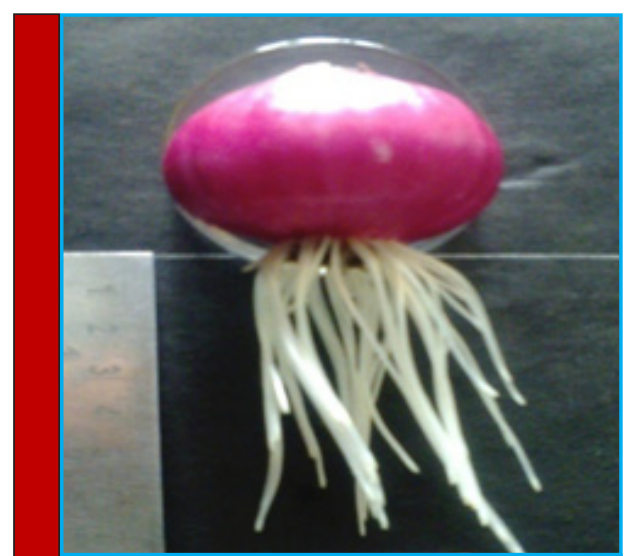

FIGURE 3. Sample of an onion bulb showing the normal root growth

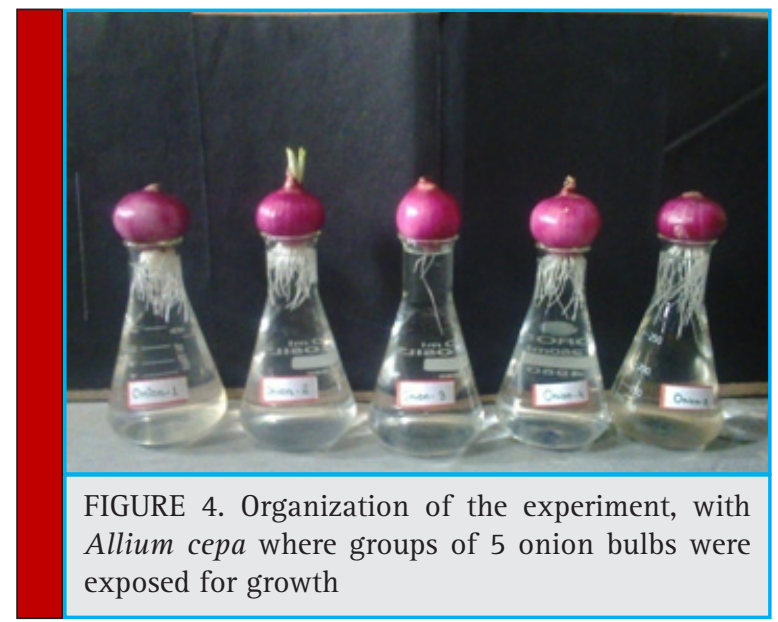

SD of the selected onion bulbs. In the root meristems of the negative control, the percentage of aberrant cells

\begin{tabular}{|c|c|c|c|c|c|c|c|}
\hline \multirow{3}{*}{ Treatment gropus } & \multirow{3}{*}{ Concentration } & \multicolumn{6}{|c|}{ Root length in different time interval (mean \pm std.error) } \\
\hline & & \multicolumn{3}{|c|}{ Before treatment } & \multicolumn{3}{|c|}{ After treatment } \\
\hline & & $24 \mathrm{hrs}$ & $48 \mathrm{hrs}$ & 72hrs & $24 \mathrm{hrs}$ & $48 \mathrm{hrs}$ & $72 \mathrm{hrs}$ \\
\hline Control & --- & $0.16 \pm 0.045$ & $0.8 \pm 0.078$ & $3.67 \pm 0.136$ & $6.04 \pm 1.34$ & $7.57 \pm 1.44$ & $9.04 \pm 1.65$ \\
\hline Positive Control (MMC) & $2 \mathrm{mg} / \mathrm{lit}$ & $1.64 \pm 0.22$ & $2.97 \pm 0.37$ & $4.57 \pm 0.93$ & $5.54 \pm 0.59$ & $5.7 \pm 0.55$ & $5.77 \pm 0.62$ \\
\hline \multirow{3}{*}{ Paper Mill Effluent } & $10 \%$ & $0.26 \pm 0.075$ & $1.1 \pm 0.129$ & $4.44 \pm 0.062$ & $5.03^{* * * *} \pm 0.045$ & $5.13^{* * * *} \pm 0.045$ & $6.1^{* * *} \pm 0.107$ \\
\hline & $25 \%$ & $0.2 \pm 0.068$ & $1.23 \pm 0.091$ & $4.06 \pm 0.39$ & $5.05^{* * * *}{ }_{ \pm} 0.35$ & $4.86^{* * * *} \pm 0.349$ & $5.36 \pm 0.286$ \\
\hline & $50 \%$ & $0.36 \pm 0.045$ & $1.67 \pm 0.169$ & $2.96 \pm 0.223$ & $3.9^{* * * *} \pm 0.223$ & $4.53^{* * * *} \pm 0.075$ & $4.93 \pm 0.062$ \\
\hline \multirow{3}{*}{ Domestic Sewage } & $10 \%$ & $0.23 \pm 0.062$ & $1.93 \pm 0.219$ & $4.13 \pm 0.164$ & $4.8^{* * * *} \pm 0.165$ & $5.3^{* * * *} \pm 0.165$ & $5.7 \pm 0.186$ \\
\hline & $25 \%$ & $0.13 \pm 0.068$ & $1.23 \pm 0.091$ & $4.33 \pm 0.169$ & $4.9^{* * * *} \pm 0.181$ & $5.3^{* * * *} \pm 0.181$ & $5.66 \pm 0.198$ \\
\hline & $50 \%$ & $0.35 \pm 0.062$ & $1.73 \pm 0.248$ & $3.73 \pm 0.091$ & $3.96^{* * * * *} \pm 0.075$ & $4.3(( \pm \pm 0.029$ & $4.5 \pm 0.029$ \\
\hline
\end{tabular}




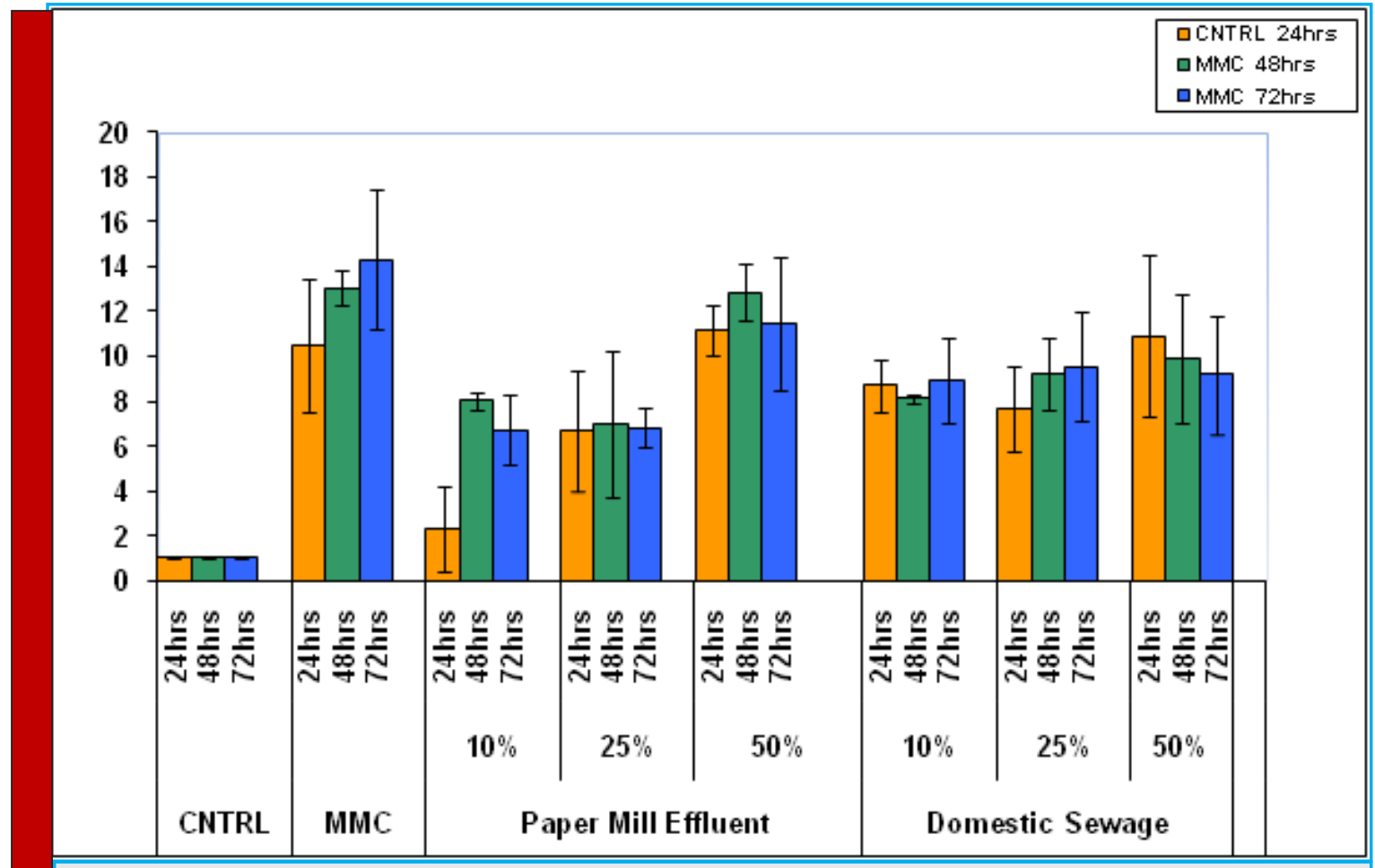

FIGURE 5. Graphical representation of various types of meiotic abnormalities after treatment with different concentrations of Paper Mill Effluent and Domestic Sewage.

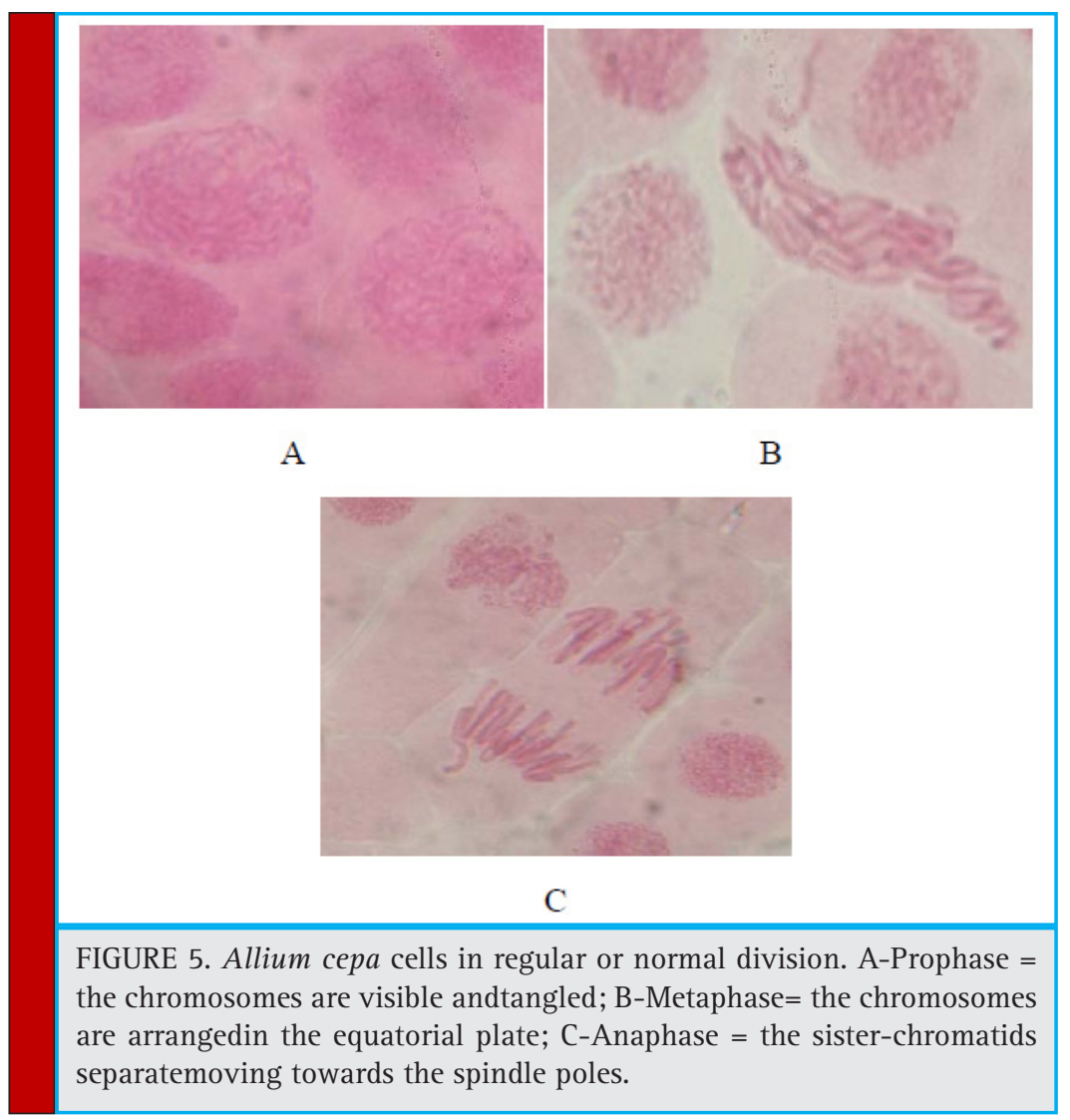




\begin{tabular}{|c|c|c|c|c|c|c|c|c|c|c|c|c|c|c|c|c|c|c|c|c|c|c|c|c|c|}
\hline \multirow{16}{*}{ 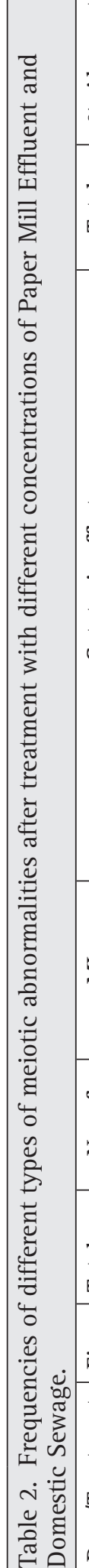 } & \multirow{2}{*}{\multicolumn{2}{|c|}{ 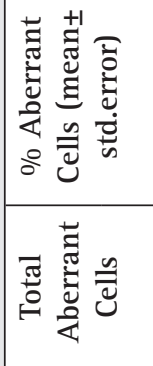 }} & $\begin{array}{l}0 \\
0 \\
0 \\
0 \\
y\end{array}$ & & & 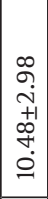 & & & 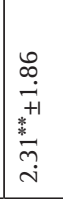 & 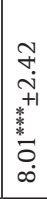 & 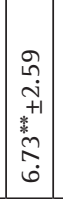 & 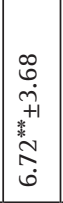 & & 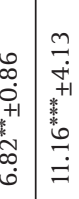 & 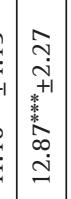 & 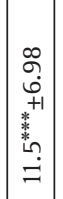 & \multicolumn{2}{|c|}{ 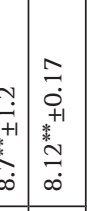 } & 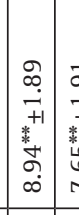 & \multicolumn{2}{|c|}{ 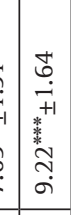 } & 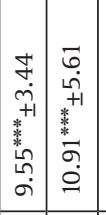 & \multicolumn{2}{|c|}{ 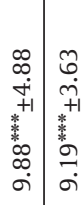 } & 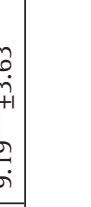 \\
\hline & & & 0 & g & 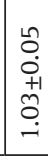 & $\stackrel{\sim}{\sim}$ & 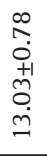 & 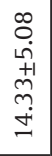 & $\overrightarrow{\tilde{v}}$ & f & 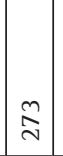 & 苍 & 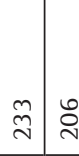 & $\begin{array}{c}\tilde{N}^{\circ} \\
\text { m }\end{array}$ & $\begin{array}{l}\infty \\
q \\
q\end{array}$ & 㤐 & 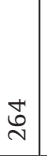 & $\stackrel{\infty}{\sim}$ & 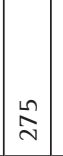 & 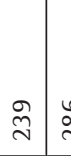 & 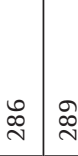 & 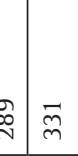 & $\bar{m}$ & 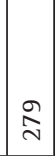 & \\
\hline & & & 0 & 。 & - & N & 产 & F & $\vec{n}$ & 类 & 悉 & \& & \begin{tabular}{l|l}
$\curvearrowright$ & $*$ \\
$\sim$
\end{tabular} & $\stackrel{*}{*} \quad \infty$ & 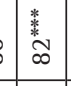 & 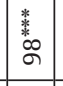 & $m$ & $m$ & 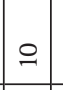 & 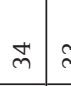 & $\stackrel{m}{m}$ & $\dot{m}$ & F & $\begin{array}{l}\text { 葛 } \\
\end{array}$ & 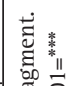 \\
\hline & & U & 0 & 0 & - & \llcorner & $\overrightarrow{\mathrm{N}}$ & $\stackrel{\infty}{=}$ & $\approx$ & $\stackrel{*}{=}$ & a & $\equiv$ & $\because \approx$ & 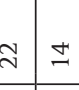 & i & in & \llcorner & 6 & $\cong$ & 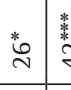 & 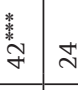 & ث & 偻 & 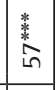 & \\
\hline & & ชै & 0 & 0 & 0 & $\sim$ & $\stackrel{0}{2}$ & $\tilde{\nu}$ & $\eta$ & $m$ & $\sigma$ & $\cong$ & ì & $\vec{\sim}=$ & $\stackrel{ \pm}{\sim}$ & 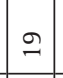 & $\stackrel{\Delta}{\Delta}$ & m & $\stackrel{m}{m}$ & 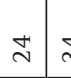 & 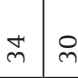 & $\begin{array}{lll}0 & \pi \\
\end{array}$ & $\vec{m}$ & i & \\
\hline & 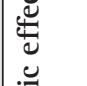 & $z_{m}$ & 0 & $\circ$ & 0 & $m$ & 0 & $\infty$ & $\cong$ & $\theta$ & $\because$ & $\stackrel{2}{\approx}$ & $\stackrel{\infty}{\rightarrow} \stackrel{i}{\sim}$ & i & $\stackrel{\leftrightarrow}{\sim}$ & $\begin{array}{l}\text { 絭 } \\
m\end{array}$ & - & - & $m$ & $\infty$ & 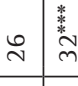 & c & $\stackrel{*}{\sim}$ & $\begin{array}{l}\text { 悉 } \\
\text { m }\end{array}$ & \\
\hline & 总 & S & 0 & 0 & 0 & 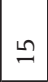 & 6 & $\infty$ & 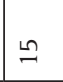 & 6 & $a$ & i & 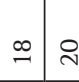 & $\stackrel{\sim}{i}$ & m & g & 皇 & 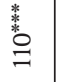 & $\mid \begin{array}{l}6 \\
\stackrel{6}{6} \\
=\end{array}$ & 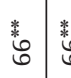 & 聕㐘 & 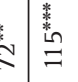 & 事 & 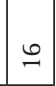 & \\
\hline & & zis & 0 & 은 & 0 & $\infty$ & $\stackrel{\infty}{-}$ & ป & 美 & 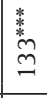 & 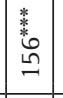 & 桸 & 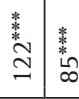 & 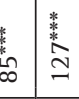 & 悉 & 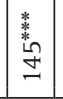 & 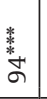 & 类 & 窭 & : & \begin{tabular}{l|l}
$*$ \\
\end{tabular} & $6 \stackrel{3}{*}$ & 苔 & $\ddot{m}$ & \\
\hline & & 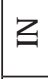 & 0 & $\circ$ & 0 & N & $\stackrel{\infty}{\sigma}$ & $\bar{\Xi}$ & ९े & 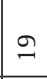 & $\stackrel{0}{0}$ & 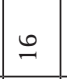 & $i=$ & 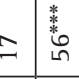 & 墨 & \begin{tabular}{|l} 
糙 \\
$\infty$
\end{tabular} & 0 & 0 & - & $\stackrel{2}{2}$ & $\stackrel{\sim}{\sim})$ & $\begin{array}{ll}0 & n\end{array}$ & $\stackrel{\text { in }}{\stackrel{*}{m}}$ & $\begin{array}{l}\text { 类 } \\
\text { 亡n } \\
\end{array}$ & \\
\hline & & 㥈 & 0 & 。 & 。 & $\stackrel{\sim}{\sim}$ & $\stackrel{N}{N}$ & $\stackrel{\infty}{\sim}$ & 0 & 0 & 0 & 0 & \begin{tabular}{l|l}
0 & 0 \\
\end{tabular} & $\begin{array}{lll}0 & 0\end{array}$ & $m$ & 意 & 0 & $\circ$ & 0 & 0 & \begin{tabular}{l|l}
0 & 0 \\
\end{tabular} & \begin{tabular}{l|l}
0 \\
\end{tabular} & - & + & \\
\hline & 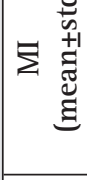 & & 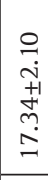 & 0 & $\circ$ & 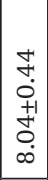 & 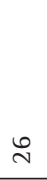 & $\tilde{m}$ & 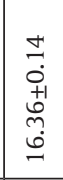 & 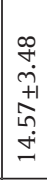 & 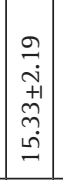 & 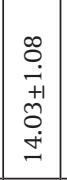 & & 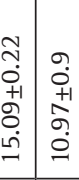 & 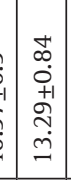 & 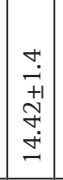 & 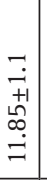 & 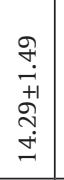 & 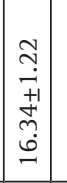 & 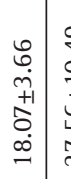 & 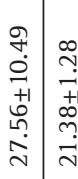 & 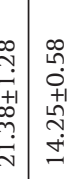 & 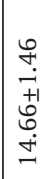 & 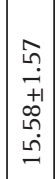 & 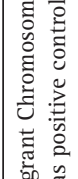 \\
\hline & 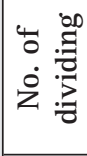 & & 羿 & & 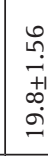 & 文 & $\begin{array}{c}0 \\
0 \\
0 \\
0+1 \\
0+1 \\
0 \\
0\end{array}$ & 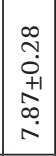 & $\vec{g}$ & b & $\widetilde{f}$ & శ్ & \begin{tabular}{lll}
$\vec{b}$ & \multirow{2}{*}{} \\
\end{tabular} & $\begin{array}{l}0 \\
b\end{array}$ & 呆 & $\hat{\vec{\gamma}}$ & 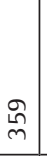 & 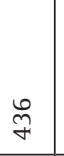 & $\tilde{n}$ & \begin{tabular}{l|l}
$\vec{n}$ & $\hat{3}$
\end{tabular} & 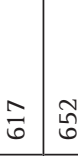 & 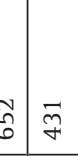 & 豙 & $\stackrel{\Re}{f}$ & 童 \\
\hline & 褐 & & 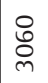 & $\frac{9}{6}$ & స్ర & $\mid \begin{array}{l}0 \\
0 \\
m \\
m\end{array}$ & त̇ & 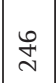 & $\overrightarrow{\overrightarrow{0}}$ & 问 & 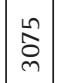 & \begin{tabular}{|l}
$\overrightarrow{0}$ \\
$\vec{n}$
\end{tabular} & 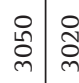 & : & $\begin{array}{l}\hat{o} \\
\vdots \\
0\end{array}$ & $\mid \begin{array}{c}\hat{\tilde{o}} \\
\tilde{\rho}\end{array}$ & $\overrightarrow{\tilde{\rho}}$ & $\overrightarrow{\tilde{\sigma}}$ & $\mid \begin{array}{l}\hat{d} \\
\text { d }\end{array}$ & 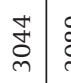 & 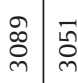 & 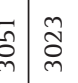 & 足 & 导 & \\
\hline & 芦节 & & $\sim$ & & 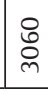 & I & बे & $\stackrel{\vartheta}{\bar{m}}$ & 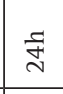 & 韋 & तี & 胥 & 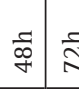 & సี & $\mid \begin{array}{c}\vec{s} \\
\stackrel{s}{a}\end{array}$ & 종 & ‡্ & $\begin{array}{l}\overrightarrow{\mathrm{o}} \\
\stackrel{\alpha}{\alpha}\end{array}$ & సี & 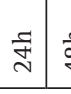 & 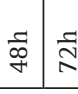 & Iี & 离 & స्ञ & \\
\hline & 苞 & & & & & & & & & $\stackrel{9}{9}$ & & & ڤ્ํํ & & 亏ें & & & $\stackrel{\circ}{\circ}$ & & & 今ें & & ఫิ & & \\
\hline & 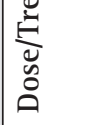 & & & & & & & & & & & & & & & & & & & & & & & & \\
\hline
\end{tabular}




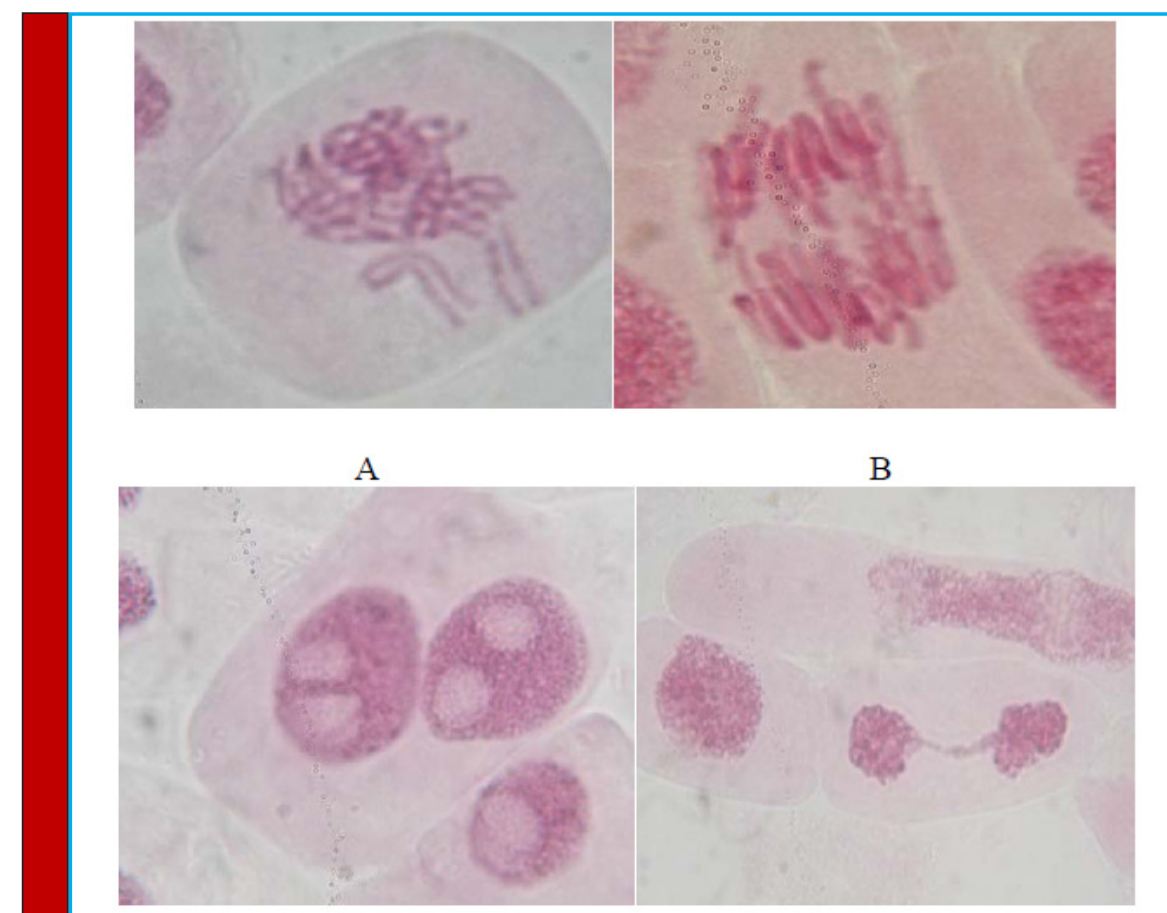

$\mathrm{C}$ $\mathrm{D}$

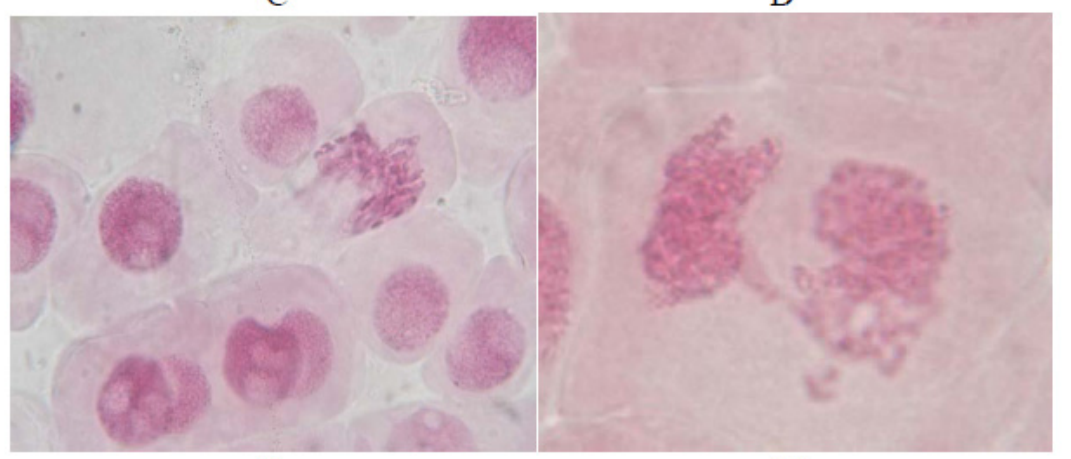

$\mathrm{E}$

F

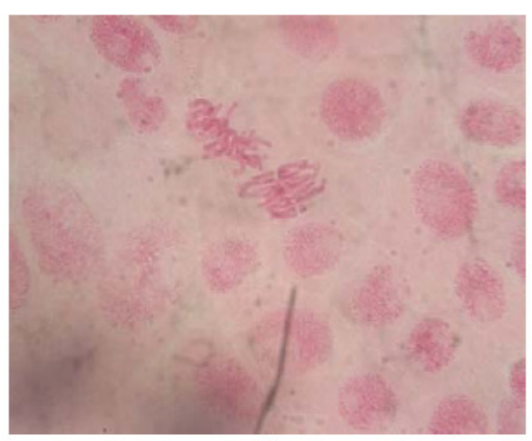

G

FIGURE 6. Allium cepa meristematic cells showing the alterations due to the action of industrial effluent and domestic sewage; A-irregular metaphase, with unorganized chromosome, alsoknown as C-metaphase, showing chromosomes with no orientation on the equatorial plate; B-irregular anaphase,with anaphasicmicrobridges; C-irregular cell, binucleate, with an elliptical aspect; D-telophase bridge; E-cell with adherent or damaged nucleus, F-irregular cell; G-metaphase with numerical alteration, due to duplication of the number of Chromosomes. 


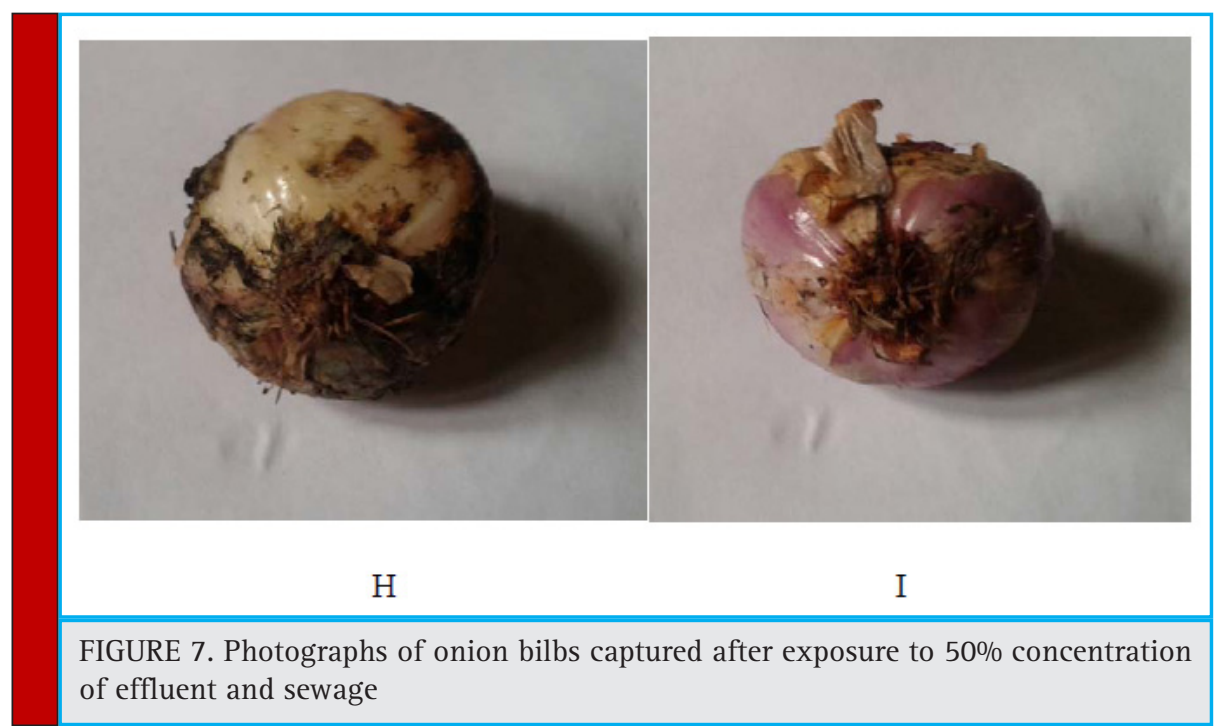

represents an avg. of 1.03 which was recorded as 8.34 and 10.09 in effluent and sewages.

Results obtained in our study showed that sewage and effluent induced chromosomal aberration through interactions with DNA and proteins leading to chromosome stickiness, mitotic disturbances and cell damage. Present finding is similar to the results of Odeigah et al. (1997b) in which roots of Allium cepa exposed to effluent from industrial wastes resulted to cells with chromosomal aberration. It would be beneficial to apply $A$. cepa chromosomal assay as a tool for monitoring the genotoxic effects of industrial effluent and wastewaters thereby providing information on the need for environmental managers to further subject treated industrial effluent to Toxicity Identification Evaluation and Toxicity Reduction Evaluation before they are finally discharged. This will enable proper chemical analysis of industrial effluent in order to identify the constituent that is really genotoxic and its prompt removal from the effluent before discharge.

\section{CONCLUSION}

According to the environmental campaign organization WWF," Pollution from toxic chemicals threatens life on this planet. Every ocean and every continent, from the tropics to the once-pristine polar regions, is contaminated. Clearly the problems associated with water pollution can disrupt life on our planet to a great extent. Increasing population and industrialization and direct exposure of sewage in water bodies has resulted in production of huge amount of liquid waste which ultimately finds its way to water bodies like rivers, lakes, streams and oceans. These effluents and sewages if provided to plants, animals and human for consumption, can lead to spreading of different types of hazardous diseases. Hence, it becomes mandatory to analyze the level of pollution caused to water and its flora and fauna.

We can conclude from our investigation that Allium cepa test is an excellent biomarker of chromosomal aberrations and has occupied an important place for the prevention and prediction of environmental impact that will be caused by the exposure of effluents and sewages. The above findings revealed the fact that industrial effluent and domestic sewage have almost equivalent capabilities to cause harmful damages to the water bodies. Life is ultimately about choices and so is pollution. We can live with sewage strewn beaches, dead rivers, and consume fish that are too poisonous for us to eat. Or we can work together to keep the environment clean so that plants, animals and people who depend on it remains healthy. It is ultimately up to us to be informed, responsible and involved when it comes to the problem we face with our water. We must become familiar with our water resources and learn about ways for disposing harmful household wastes so that they do not end up in sewage treatment plants that can't handle them or landfills not designed to receive hazardous materials. Hence proper measures should to be taken for the management of water quality and the aquatic life.

\section{ACKNOWLEDGEMENTS}

We are grateful to UGC for providing fellowship to RP and BR.

\section{REFERENCES}

A.A. Bakari, O.A. Alabi, A.M. Gbadebo, O.I. Ogunsuyi and C.G. Alimba, (2017) In vivo cytogenotoxicity and oxidative stress 
induced by electronic waste leachate and contaminated well water Challenges, vol. 4, pp.169-187

A. Gill and M.I.S. Saggoo (2010) Mutagenic Potential and Nutritive Quality of Turnip Plants Raised over Chromium Amended Soils International Journal of Botany., vol . 6, pp. 127-131

A.H. Siddiqui, S. Tabrez, and M. Ahmed (2011) Validation of Plant based bioassays for the toxicity testing of Indian waters, Environ Monitor Assess., vol. 179,1-4, pp. 241-253, ISSN 1573-2959

A.K. Sharma, and A.S Dphil (2012) Chromosome techniques, Fakenham press ltd, Norfolk. Pp. 95-100

A. Prasad, and K.V.B. Rao, (2010) Physico Chemical Analysis of Textile Effluent and Decolorization of Textile Azo Dye by Bacillus endophyticus Strain VITABR 13, "The IIOAB Journal., vol. 2, pp. 55-62

A.S Margiassi, M.S. Goldbergs, A.J. Wheeler, C. Plante, M.F. Valois, G. Mallach, (2014) Associations between perreneal exposure to air pollutants and lung function tests and cardio vascular indices among children with asthma living near industrial complex and petroleum refineries Environ. Res.,132, pp.38-45

A.W. Amin (2008) Cytotoxicity testing of sewage water treatment using Allium cepa chromosome aberration assay Pakistan Journal of Biological Sciences. 5(2): pp.184 -188

Amin AW and M.M. Muzahid (2009) Cytogenetic effect of sea water irrigation on irradiated wheat grains Egyptian Journal of Genetics and Cytology. 29: pp. 199 -213

Zegura B F. Heath, A. Černoša, M. Filipic (2009) Combination of in vitro bioassays for the determination of cytotoxic and genotoxic potential of wastewater, surface water and drinking water samples Chemosphere, 75, PP. 1453-60

Michael C, and G.C. Odeigah (2009) The genotoxicity effect of sewage effluent on Allium cepa Report and Opinion., vol. 1(6), pp. 36-41

Marin-Morales DM (2009) Allium cepa test in environmental monitoring: a review on its application Mutat Res; 682: pp. $71-81$

Schieltz ES A.A. Litomjua, E. Melen (2017) (Effects Of Long term exposure to Traffic related Air Pollution on Lung function in children Curr. Allergy Ashthma Rep. vol. 41: pp.1-13

Friskesjo G (2011) The Allium test, an alternative in environmental studies; the relative toxicity of metal ions Mutation Research. 197, pp. 243-280

Zaiad, GM (2010) Physico-chemical Analysis of Soils in AlKhums city, Libya Journal of Applied Sciences Research, vol. 6, pp. 924-929

Pink HD (2006) Heavy metals, Investing in Tomorrow's Liquid Gold, 3748, 2006.

Grover IS, and S. Kaur (2009) Genotoxicity of wastewater samples from sewage and industrial effluent detected by the Allium root anaphase aberration and micronucleus assays Mutat. Res., 426, pp. 183-188, ISSN 0027-5107

Krishnamurthi K, S.S. Devi, J.G. Hengstler, M. Hermes, K. Kumar, D. Dutta, S.M. Vannan, T.S. Subin, R.R. Yadav, T. Chakrabarti, (2008) Archieves of Toxicilogy. , vol. 82, pp. 965-971
Souza LFB H.D. Laughinghouse, T. Pastori, M. Tedesco, A.W. Kuhn, T.S. Canto-Dorow, S.B. Tedesco (2010) Genotoxicity potential of aqueous extract of Artemisisa verlotorum on the cell cycle of Allium cepa, Int. J. Environ Stud., vol. 67,6,pp. 871-877, ISSN 00207233

Anacleto, LR M.M. Roberto, M.A. Marin-Morales (2017) Toxicological effects of the waste of the sugarcane industry, used as agricultural fertilizer, on the test system Allium cepa Chemosphere. vol.173, pp. 31-42

Sik, L 0. Acar, and C. Aki, (2009) Genotoxic Effect of Industrial Waste water on Allium cepa African Journal of Biotechnology., vol. 8, pp. 1919-1923

Saavedera, MA and P. Arambari (1989) Use of sewage water for irrigation of olive trees Cytologia. 54: pp. 489-492

Bennet MD (1997) Heterochromatin, aberrant endosperm nuclei and grain shriveling in wheat-rye genotypes. Heredity. 39: pp. 411-418

Kataeva M , V. Kotseruba, N. Terekhina, N. Kutlunina, A. Beljaeva (2010) Allium Root Micronuclues Test on the Genotoxicity of Soil rosamples Contaminated with Heavy Metals World Applied Science Journal., vol . 17, pp. 992-1000

Koçyigit M and N. Özhatay, A contribution to the genus Allium L. (Sect. Codonoprasum) in Turkey. Turkish Journal of Botany, 34, pp. 391-395, 2010.

Kurt OK, J. Zhang, K.E. Pinkertan, (2016) Pulmonary health effects of water pollution Pulm. Med., 22, pp. 138-43

Kolawole OM, and T.O. Sunmonu (2010) Effect of Wasterwater Treated with Metanolic Bark Extract of Bridelia ferruginea Benth on Rat Kdiney and Liver, Journal of Applied Sciences in Environmental Sciences., vol. 5, pp. 55-64

Gupta P, N. Mathur, P. Bhatnagar, P. Nagar, and S. Srivastava (2009) Genotoxicity Evaluation of Hospitality Waste water Ecotoxicology and Environment Safety, vol. 72, pp. 1925-32

Odeigah PGC, O. Nurudeen, O Amund (1997) Genotoxicity of oil field waste water in Nigeria Hereditas. 126, pp.161-167

Odeigah PGC, J. Makinwa, B. Lawal and R. Oyeniyi (1997b) Genotoxicity screening of leachates from solid industrial waste evaluated Allium test ATLA. 25, pp. 311-321

Mannucci, PM S. Harasi, I. Martinelli, M. Franchini (2015) Effects on health of air pollution: A narrative review Intern. Emerg. Med. Springer Milan, 10: pp. 657-662

Nirel R, N. Maimon, E. Fireman, S. Agami, A. Eyal, A. Peretz. (2015) Respiratory Hospitalization of children living near a hazardous industrial site adjusted for prevalent dust: A case central study Int. Journal of Hyg. Environ. Health. 218: pp. 273-279

Alrumman, SA A.F. El-kott, M.A. Kehsk, "Water pollution: Source and treatment", American journal of Environmental Engineering. 6(3), PP. 88-98, 2016.

Bibi S, R.L. Khan, R. Nazir (2016) Heavy metals in drinking water of Lakki Marwat District, KPK, Pakistan World applied sciences journal, 34(1), PP.15-19

Wong, SL A.L. Coates, T.To, (2016) Exposure to industrial air pollutant emission and lung functionin children: Canadian 
Health Measures Survey, 2007 to 2011. Health Reports. 27: pp. 3-9

Rajkumar S R, P. Velmurugan, K. Shanti, P.M. Ayyasamy, and P. Lakshmanaperumalsamy (2004) Water Quality of Kodaikanal Lake, Tamilnadu in relation to physico-chemical and bacteriological characteristics Capital Publishing Company, Lake., pp. 339-346

Suriyanarayanan S., G. Jessen, L. Divya, and S. Balasubramanian, (2012) Effect of waste paper based paper industry effluents on the growth of tree seedlings, J. Environ. Res. Develop., vol. 7(2A), pp. 1117-1126
Oyeniyi T, C. Latunji (2012) Industrial Effluent Induced Abnormal Sperm Cells in mice (Mus musculus) New York Science Journal vol. 5, pp. 60-64

Smaka-Kinel V, P. Stegner, M Lovka, and M. J, Toman (2013) The evaluation of waste, surface and ground water quality using the Allium test procedure, Mutation Research. 368: PP.177-179

Ahmed ZH , and S.N. Suhanija (2013) Physiological and biochemical responses of Malaysian red alga, Gracilaria manilaensis treated with copper, lead, mercury, J. Environ. Res. Develop., vol.7(3), pp. 1246-1253 